\title{
Problèmes rencontrés lors de la datation par thermoluminescence
} d'un four de potier

Hélène Valladas

\section{Citer ce document / Cite this document :}

Valladas Hélène. Problèmes rencontrés lors de la datation par thermoluminescence d'un four de potier. In: Revue d'Archéométrie, n¹, 1977. pp. 127-133;

doi : https://doi.org/10.3406/arsci.1977.1086

https://www.persee.fr/doc/arsci_0399-1237_1977_num_1_1_1086

Fichier pdf généré le 07/01/2019 


\section{PROBLEMES RENCONTRES LORS DE LA DATATION PAR THERMOLUMINESCENCE}

D'UN FOUR DE POTIER

par

Hélène VALLADAS

Centre des Faibles Radioactivités

Laboratoire mixte C.N.R.S - C.E.A

9II90 - Gif-sur-Yvette

\section{I - INTRODUCTION}

Le four Grévin découvert fortuitement au cours de 1'année 1974 dans le village de Savignies (Beauvaisis) a été fouillé en sauvetage par le

:usēe des Arts et Traditions Populaires. J.Y. HAMEL a fait I'étude typologique des grès trouvés à l'intérieur du four. Cette étude suggère qu'il fait partie des premiers fours à grès du Beauvaisis (2) d'où l'intérêt de sa datation. Elle a été faite de façon satisfaisante par l'équipe de E. THELLIER (1) en utilisant la méthode de l'archéomagnétisme. La date trouvée est 1380 +25 ans. La datation par thermoluminescence avait donc surtout pour nous un intérêt méthodologique. Nous nous bornerons donc dans cet exposé à ce qui nous a paru le plus utile à signaler (2).

Nous avons utilisé des prélèvements de la paroi contenant des grains de quartz en abondance. Nous avons donc affaire à une application de la méthode des inclusions développée par S.J. FLENING (1968), cependant nous avons rencontré des difficultés peu fréquentes dans la datation des céramiques. En effet :

- la paroi du four était crevassée, très poreuse aux eaux de ruissellement d'oì une contamination de nos échantillons par des grains de quartz venus du sol environnant.

- Ie four Grévin ayant produit des grès, a été amené à des températures excédant $1100^{\circ}$; les grains de quartz chauffés au contact d'une argile très ferrugineuse se sont altérés en surface : leurs propriétés étaient assez différentes de celles de quartz trouvés habituellement dans les céramiques.

Dans ce qui suit, nous allons décrire succintement :

- Ia méthode utilisée pour éliminer les grains de quartz entrainés par les eaux de ruissellement dans la paroi du four. La détermination de la dose archéologique;

- les propriétés de la thermoluminescence des grains de quartz et la détermination de leur dose $\alpha$ effective;

- Ia mesure de la concentration en eau de nos échantillons.

(1) Communication privée.

(2) La datation par thermoluminescence du four Grévin est contenue dans un iémoire de mâtrise d'archéologie (J.Y. HAMEL, E. VALLADAS, 1976) (voir bibliographie). 
II - PREPARATION DES ECHANTILLONS : SEPARATION NAGNETIQIIE.

\section{Mesures préliminaires}

Les premières mesures effectuées sur la granulométrie 63-100 $\mu$ étaient encourageantes car elles donnaient d'emblée une dose archéologique en accord avec ce qu'on pouvait attendre pour ce four. La figure 1 représente le résultat de ces mesures en fonction de la dose du rayonnement $\beta$ d'une source de strontium 90 ajoutée à la dose archéologique. L'extrapolation de la droite obtenue $D$ donne une dose $\beta$ équivalente de 178 rads.

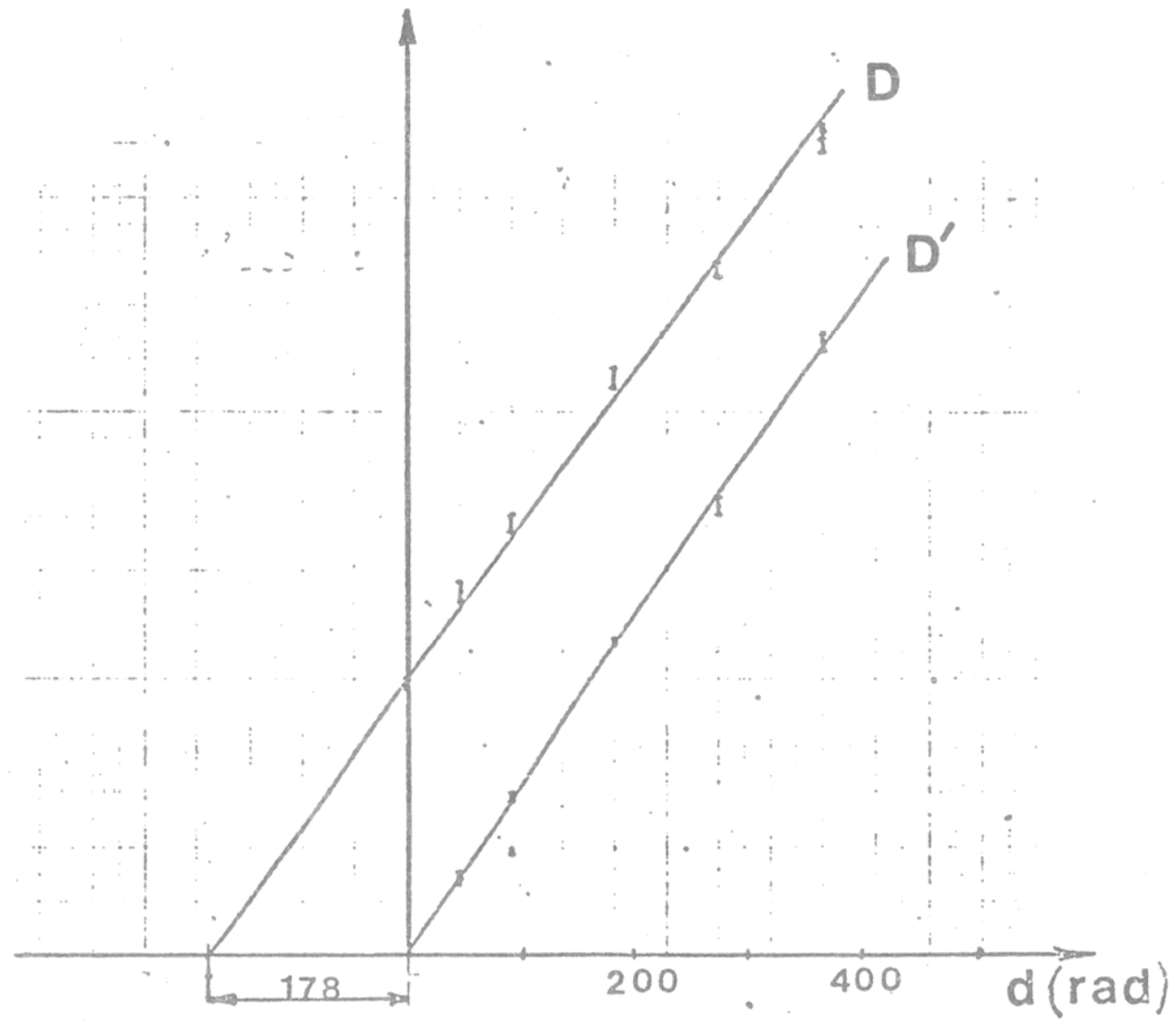

Fig.1

On peut s'assurer que la correction $\Delta$ de supralinéarité est nulle par la thermoluminescence obtenue en fonction de la dose de réirradiation appliquée à chacun des échantillons après la première chauffe. Elles sont bien représentées par une droite $D^{\prime}$ parsant par l'origine et sensiblement parallèle à la droite $D$ relative à la prerière chauffe.

Néanmoins, dans l'intervalle de température utilisé compris entre 260 et $310^{\circ}$ Cle rapport TLN/TLA (1) variait de 15 à $20 \%$ ce qui est inacceptable. En examinant les mesures, nous avons constaté leur mauvaise reproductibilité au delà de $300^{\circ} \mathrm{C}$. Sur la figure 2 nous avons représenté la courbe de TLN ( + une dose $\beta$ de 23 rads qui montre la réponse du quartz à basse température) de 3 échantillons. Le premier et le troisième montrent une thermoluminescence qui chûte au-delà de $325^{\circ}$. Le second qui a la même réponse que les deux autres à basse température a un surcrốt de thermoluminescence à haute température.

(1) TLN = thermoluminescence naturelle

TLA = thermoluminescence artificielle. 


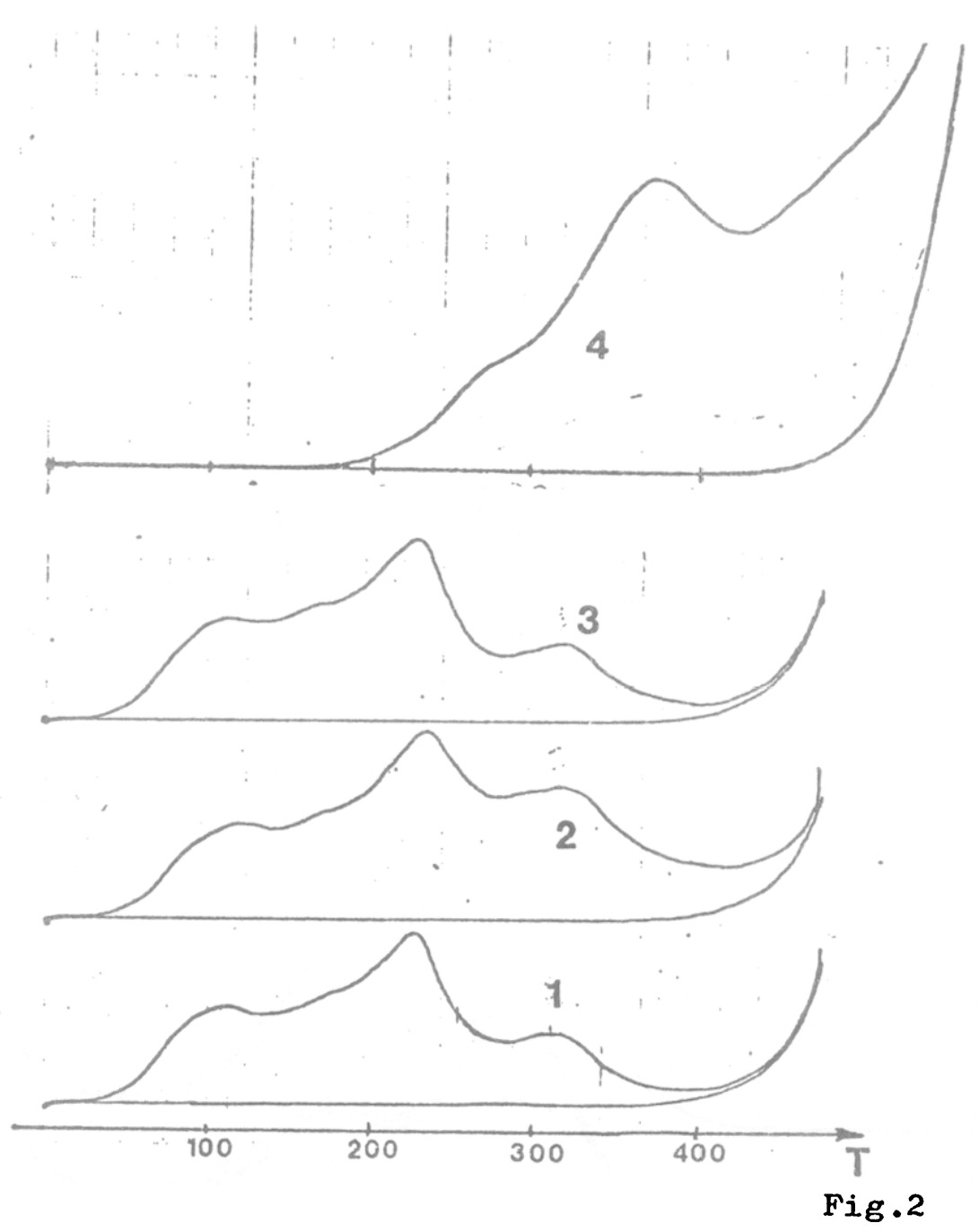

Nous avons expliqué ce manque de reproductibilité par une contamination de l'échantillon par des quartz du sol environnant. Sur la même figure la courbe (4) représente la TLN d'un mg de quartz extrait du sol environnant le four, caractérisée par une très forte émission à $375^{\circ} \mathrm{C}$. Il suffit donc de quelques grains de ce quartz pour obtenir une contribution importante de "TL géologique".

\section{Séparation magnétique}

La procédure utilisée pour éliminer la contamination du sol a été décrite par ailleurs (H. VALLADAS, 1977). Rappelons brièvement la démarche suivie :

Nous avons observé que beaucoup des grains de quartz de la paroi étaient rougeâtres. Nous avons attribué cette coloration à la présence d'une pellicule ferrugineuse développée pendant la période d'activité du four et causée par la diffusion thermique du fer de l'argile dans les grains de quartz.

Les grains de quartz d'apport extérieur ne doivent pas être ferrugineux parce qu'ils n'ont pas subi de cuisson. Nous avons cherché à utiliser un ferromagnétisme acquis par les grains de quartz cuits dans la paroi pour les différencier des gralns de quartz d'apport extérieur : le quartz pur est diamagnétique. Nous avons utilisé un séparateur isodynamique Frantz (A.PARFENOFF, C. POMEROI, J. TOURENQ, 1970) qui permet de trier les minéraux selon leur succeptibilité magnétique. Sans rappeler les conditions expérimentales de la séparation, nous allons en examiner les étapes et les résultats relatifs au quartz de la granulométrie 80-100 microns qui a pu nous servir ensuite à la détermination de l'âge du four. 
Au cours d'une première séparation, nous avons obtenu comme fraction la plus magnétique un lot composé surtout d'agglomérats très colorés représentant $60 \%$ de l'échantillon de départ (A). Nous avons fait subir une nouvelle séparation (en augmentant l'intensité) à la fraction la moins magnétique contenant surtout les grains de quartz isolés. Elle nous a donné une fraction magnétique ( $38 \%$ ) composée de grains jaunes (B) et une fraction non magnétique $(2 \%)$ de grains incolores $(C)$.

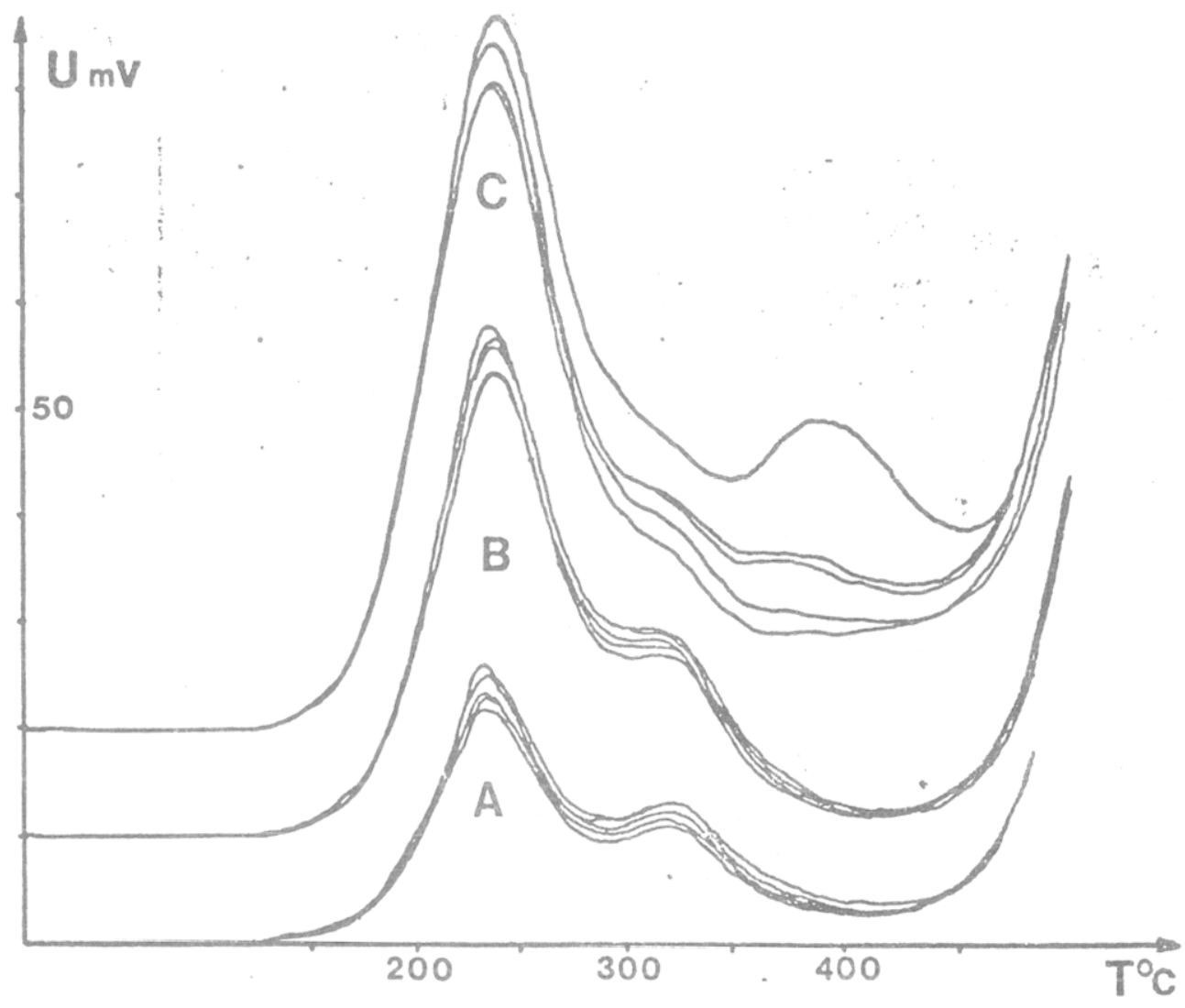

Un groupe de courbes de TLN des trois fractions $A, B, C$ est représenté sur la figure 3. Les émissions lumineuses naturelles des fractions A et B présentent le même profil en fonction de la température.

Elles ont toutes deux un maximum à 240 et $325^{\circ} \mathrm{C}$. Notons que les mesures sont bien reproductibles. Les courbes de la fraction $C$ ont les mêmes maximas à 240 et $325^{\circ}$ mais au de-là de $325^{\circ}$, l'émission lumineuse varie d'un échantillon à l'autre. Si l'on compare les courbes de la fraction $C$ à celle du quartz du sol environnant on constate que certaines ont le même maximum à $375^{\circ}$ que ce quartz. On peut alors penser que la fraction $C$ est contaminée par ce quartz (figure 2 , courbe $n^{\circ} 4$ ).

Les mesures faites sur l'échantillon $B$, issu de la séparation magnétique, nous ont donné par simple extrapolation linéaire une dose archéologique de 163 +10 rads (nous avons vu précédemment que la correction de supralinéarite était ñégligeable).

III - PROPRIETES DE LA THERMOLUMINESCENCE DES GRAINS DE QUARTZ ET DETERMINATION DE LA DOSE EFFECTIVE

Nous avons constaté au cours du décapage à l'acide fluorhydrique des grains de quartz, que ces derniers n'étaient sensibles que près de leur surface. En effet, après une attaque de cinq heures à l'acide fluorbydrique dilué à $4 \%$, le pic à $240^{\circ} \mathrm{C}$ est réduit d'un facteur 7 et le pic à $35^{\circ} 5^{\circ} \mathrm{C}$ d'un facteur 4 (figure 3 ).

Du fait que la thermoluminescence des grains est concentrée au voisinage de la surface, il découle que l'effet du rayonnement $\alpha$ se trouve grandement accru par rapport à celui du rayonnement $\beta$ qui n'agit plus comme d'ordinaire sur l'ensemble du grain. 
Pour connaltre la dose $\beta$ équivalente à la quantité de rayonnement $\alpha$ reçue par les grains, nous avons exposé successivement les échantillons à une source a et à une source $\beta$ étalonnées. Pour que cette comparaison ait un sens, il faut simuler les conditions d'irradiation d'un grain de quartz soumis aux $\alpha$ d'énergie variable provenant des sources contenues dans l'argile qui l'environne (les grains de quartz ne contiennent pas de radioéléments).

L'énergie d'un a qui atteint un grain de quartz noyé dans l'argile dépend à la fois du noyau émetteur et de sa distance au grain. Si nous supposons que les radioéléments sont répartis uniformément dans l'argile, nous pouvons calculer les flux respectifs des $\alpha$ de la famille de l'uranium et du thorium qui pénètrent dans un grain, en fonction de leur énergie.

Nous obtenons les spectres de la figure 4 (D.W. ZIMMERMAN, 1971) correspondant à $1 \mathrm{ppm}$ en uranium ou en thorium. En abscisse est représentée l'énergie des $\alpha$ et en ordonnée, le nombre $d ' \alpha$ par unité d'énergie. Nous avons mesuré la dose $\beta$ équivalente pour des énergies $d$ ' réparties dans I'intervalle des énergies concernées par les spectres de la figure 4. Pour que la mesure de la dose $\mathrm{d} \alpha^{\dot{q} q u}$ soit correcte, il faut que les grains de quartz soient disposés de façon à ne former qu'une seule couche. L'empilement des grains conduirait en effet à une sous estimation de $d \alpha^{\text {equ }}$ puisque les grains placés sous la couche ne seraient pas irradiés par les $\alpha$ mais le seraient par les $\beta$ plus pénétrarts. Les résultats des mesures des doses $\beta$ équivalentes associées à des flux d' $\alpha$ d'énergies respectivement égales à 1,$6 ; 2,7 ; 3,7 ; 4,5 ;$ et 7,2 MeV sont montrés sur la figure 4.

\section{- dose équivalente}

La dose $\beta$ équivalente moyenne reçue par an par les grains de quartz est alors donnée par la formule suivante :

$d_{\text {équ }}=\int\left(C(U) \cdot \frac{d n_{\alpha}}{d E_{\alpha}}(U)+C(T h) \frac{d n \alpha}{d E_{\alpha}}(T h)\right) S_{\alpha} d E_{\alpha}$

$C$ (U) et $C$ (Th) désignent les concentrations en $U$ et Th exprimées en ppm

et $\frac{d n_{\alpha}}{d E_{\alpha}}(U), \quad \frac{d n \alpha}{d E_{\alpha}}$ (Th) sont les flux relatifs à l'uranium et au thorium montrés par la figure 4 et $S_{\alpha}$ est le nombre de millirads par $\alpha$ et par cm2 en fonction de l'énergie des $\alpha$ montré par la figure 4 .

Le calcul de l'expression donne une dose $\beta$ équivalente aux $\propto$ de 130 $\pm 10 \mathrm{mrads} / \mathrm{an} ;$ elle est un peu plus grande que la dose $\beta$ qui est égale à $117 \mathrm{mrads} / \mathrm{an}$.

III - PROBLEME DE L'EAU

Les morceaux de paroi avaient perdu leur eau quand nous les avons eus. (La structure était gorgée d'eau en permanence). Nous avons donc cherché à estimer leur rétention en eau en les plongeant quelques jours dans un récipient d'eau : l'augmentation de poids était petite. Mais comme nous avions affaire à un matériau finement poreux, nous avons pensé que cette expérience était insuffisante. Afin d'accélérer la pénétration en eau dans les micropores de l'échantillon, nous avons fait une immersion sous vide selon la technique du porosimètre. L'augmentation de poids était de $23 \%$ de celui de I'échantillon sec.

On voit donc que la méconnaissance de la concentration en eau réelle des objets peut être une source d'erreur importante. Il est essentiel de prélever l'ensemble des échantillons au cours de la fouille et de les placer dans un récipient étanche. Toutefois cela n'est pas suffisant. En effet, puisque la concentration en eau peut varier au cours de l'année, il faut pouvoir l'esti- 

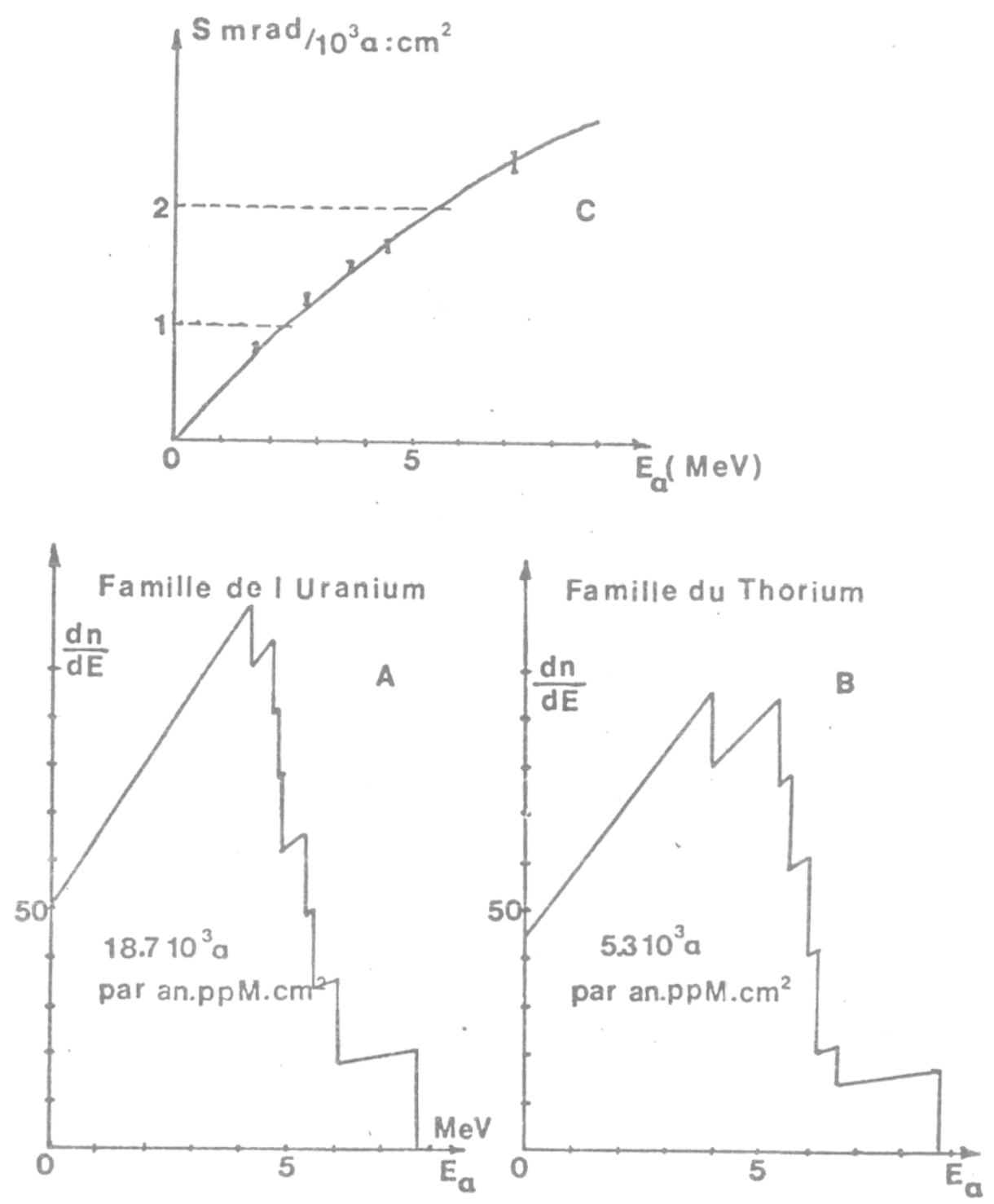

Fig. 4

En A est représenté le spectre exprimant la distribution différentielle en énergie des huit noyaux émetteurs $\alpha$ de la famille de l'uranium. En abscisse est portée l'énergie des $\alpha(\mathrm{MeV})$, en ordonnée le nombre d' $\alpha$ par $\mathrm{cm} 2$ par an et par ppm $\left(\frac{d}{d} \bar{E}\right)$. Le chiffre indiqué à l'intérieur de la courbe correspond à la surface du spectre soit le nombre total d' $\alpha$ par an, par cm2 et par ppm La courbe $B$ est relative aux sept émetteurs $\alpha$ de la famille du thorium La courbe $C$ montre la dose $\beta$ équivalente rapportée à $10^{3} / \mathrm{cm} 2$ en fonction de l'énergie des $\alpha$.

Nous avons représenté la grandeur

$S=\frac{T L(\text { irradiation } \alpha)}{T L(\text { irradiation } \beta \text { ) }} \times \frac{\text { dose } \beta \text { appliquée }}{\text { nombre total d' } \alpha / \mathrm{cm} 2}$

qui représente le nombre de millirads par $\alpha$ et par cm2 (axe des $Y$ ) elle est fonction de l'énergie des $\alpha$ (axe des $X$ ). Nous avons ajusté ensuite une courbe passant par les cinq points. 


\title{
133
}

mer a'après la profondeur d'enfouissement, la nature de la couche géologique et la circulation des eaux dans le site.

\section{CONCLUSION}

\begin{abstract}
La date que nous avons obtenue à la fin de notre tentative de datation par la thermoluminescence - dont nous venons de donner quelques aspects - est en accord avec celle de l'archéomagnétisme : $1410 \pm 70$ ans (à 20).

Un contrôle essentiel nous a fait défaut au cours de cette datation. Il aurait consisté à déterminer la dose archéologique par des méthodes indépendantes. Au départ, nous espérions appliquer les méthodes de la prédose (SJ. FLEVING) des grains fins (D.W. ZIMNERMAN, 1971) et des inclusions (gros grains). Seule la dernière a été utilisable. En l'absence de ce test de cohérence interne de la méthode : plusieurs déterminations de la dose archéologique sur des minéraux différents ou par des méthodes indépendantes, une datation par la thermoluminescence peut manquer de crédibilité.
\end{abstract}

\section{BIBLIOGRAPHIE}

AITKEN.M.J., BOWNAN.S.G.E., 1975

Thermoluminescent dating = assessment of alpha particle contribution. Archaeometry 17 (1) 129-132

FLENING.S.J., 1968

Thermoluminescence age studies in mineral inclusions separated from ancient pottery

Thermoluminescence of geological materials. Edited by D.J. Mc DOUGALL Academic Press.

FLEMING .S.J • , 1970

Thermoluminescence dating : refinement of the quartz inclusion method. Archaeometry 12 (2) 133-145

HAMEL.J.Y., VALLADAS.H., 1976

Mâ̂trise d'archéologie (PARIS I)

Etude et tentative de datation par la thermoluminescence du four Grévin de Savignies.

PARFENOFF.A., POMEROL.C. et TOURENG.J., 1970

Les minéraux en grains. Masson Ed.-Paris

VALLADAS.H., 1977

On the magnetic separation of quartz for the thermoluminescence dating of an ancient kiln Archaeometry 19 (1)

ZIMMERY AN.D.W., 1971

Thermoluminescence dating using fine grains from pottery - Archaeometry

13 (1) $29-52$. 